\title{
Super-equipartition fields in simulations of photospheric magnetoconvection
}

\author{
Paul J. Bushby ${ }^{1}$ \\ ${ }^{1}$ Department of Applied Mathematics and Theoretical Physics, University of Cambridge, \\ Wilberforce Road, Cambridge, CB3 0WA, UK \\ email: P.J.Bushby@damtp.cam.ac.uk
}

\begin{abstract}
Observations of magnetic fields in the quiet Sun indicate that kilogauss-strength fields can be found in the intergranular lanes. Since the magnetic energy of these localised features greatly exceeds estimates of the kinetic energy of the surrounding granular convection, it is difficult to see how these features could be formed simply by convective flux concentration. Idealised, high-resolution simulations of three-dimensional compressible magnetoconvection are used to investigate the formation of these features numerically. Initially we take a fully developed non-magnetic convective state into which we insert a weak, uniform, vertical magnetic field. Magnetic flux is rapidly swept into the convective downflows, where it is concentrated into localised regions. As the field strength within these regions becomes dynamically significant, the high magnetic pressure leads to partial evacuation (via the convective downflows). Provided that the magnetic Reynolds number is large enough, the strength of the resulting magnetic fields significantly exceeds the (so called) "equipartition" value, with the dynamical effects of the surrounding convection playing an important role in confining these magnetic features to localised regions. These results can be related to the well-known convective collapse instability, although there are some important differences between the two models.
\end{abstract}

Keywords. Convection, magnetohydrodynamics: MHD, Sun: magnetic fields, Sun: photosphere

\section{Introduction}

High resolution observations of the solar surface have shed new light upon the complex interactions between photospheric magnetic fields and the surrounding granular convection. In the quiet Sun, magnetic flux is confined primarily to the intergranular lanes (Lin \& Rimmele 1999), where it forms localised (often "point-like") magnetic structures. Since typical magnetic field strengths in these regions can often exceed a kilogauss, the magnetic energy density of these localised flux concentrations greatly exceeds estimates of the kinetic energy density of the surrounding granular convection. Numerical simulations can help us to address the question of whether or not convective motions alone can amplify magnetic fields to these observed super-equipartition field strengths. In order to make detailed comparisons between numerical simulations and photospheric observations, it is necessary to include complex physical effects such as radiative transfer (Grossmann-Doerth, Schüssler \& Steiner 1998, Vögler et al. 2005, Stein \& Nordlund 2006). An alternative approach is to consider more idealised models of photospheric magnetoconvection (see, e.g., Weiss, Proctor \& Brownjohn 2002, Bushby \& Houghton 2005), which focus entirely upon the interactions between convection and magnetic fields and lend themselves more easily to surveys of parameter space. In this paper, we adopt an idealised model of this type in order to study magnetic field intensification in the quiet Sun. 

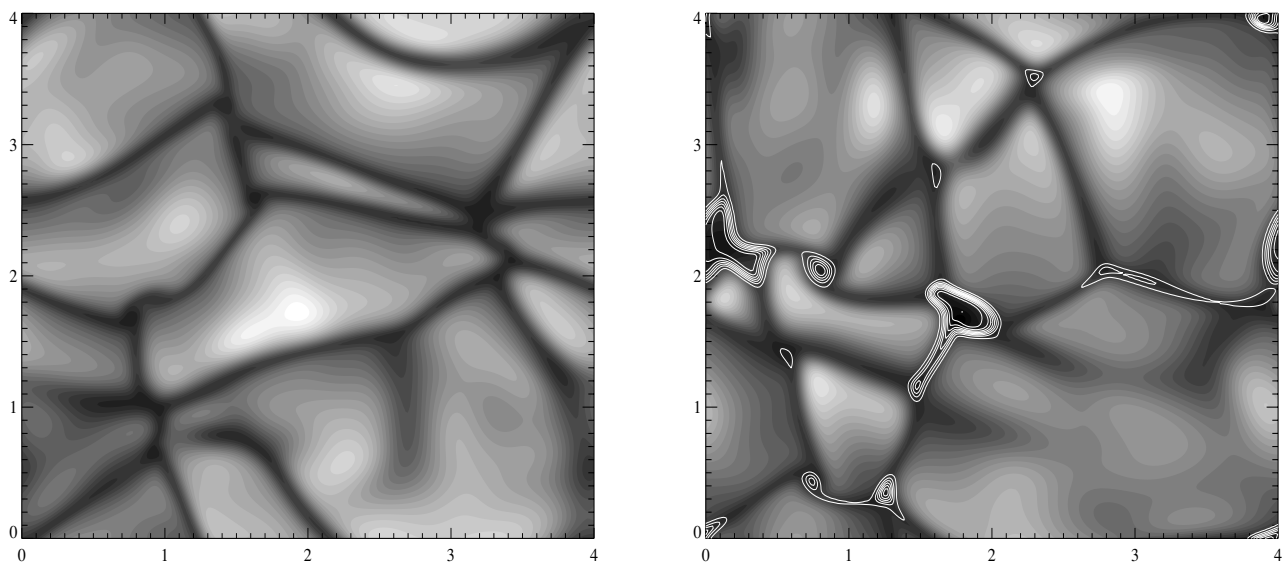

Figure 1. The temperature distribution in a horizontal layer just below the upper surface of the computational domain, for the initial (non-magnetic) state (left) and at a later time, after the magnetic field has been added (right). The white contours in the right hand plot show the vertical component of the magnetic field (which accumulates in the convective downflows).

\section{Model}

We solve the equations of compressible magnetoconvection in a horizontally-periodic, three-dimensional $(4 \times 4 \times 1)$ Cartesian domain. High resolution calculations enable us to accurately resolve features at high magnetic Reynolds numbers - the computational grid has 256 points in each horizontal direction and 160 points vertically. Full details of the non-dimensionalised equations, parameter definitions and boundary conditions can be found in Bushby \& Houghton (2005). In this calculation, we consider convection in a highly stratified $(m=1)$ polytropic layer, in which the temperature and density both vary by approximately one order of magnitude across the layer. The Prandtl number is fixed at unity, and we consider convection at high Rayleigh number $\left(R=4.0 \times 10^{5}\right)$. In order to investigate the dependence upon the magnetic Reynolds number $\left(R_{m}\right)$, we have carried out a series of calculations for different values of the magnetic diffusivity (paper in preparation). However, in this paper, we restrict attention to a single diffusivity value - using the definitions given in Bushby \& Houghton (2005), this diffusivity value corresponds to a magnetic to thermal diffusivity ratio of $\zeta=0.2$.

\section{Results}

The initial state for this calculation is a snapshot of fully evolved (non-magnetic) granular convection (see figure 1). We insert a weak, uniform, vertical magnetic field into this convective state - the strength of this field is chosen so that the (total) initial magnetic energy is only $0.1 \%$ of the initial kinetic energy. Magnetic flux is expelled from the convective upflows and forms localised structures in the narrow downflows at the edges of the granular pattern (see figure 1). As these flux concentrations form, the magnetic field becomes dynamically significant, and the high magnetic pressure leads to partial evacuation. As plasma is forced out of these concentrated regions of magnetic flux, the associated magnetic fields can be amplified to super-equipartition field strengths. For the high $R_{m}$ solution shown in figure 1 (where $R_{m} \approx 120$ ), the peak magnetic energy density is almost a factor of three larger than the peak kinetic energy density of the surrounding granular convection - large values of $R_{m}$ lead to stronger fields. Once these magnetic flux features have formed, the sum of the gas pressure plus the 

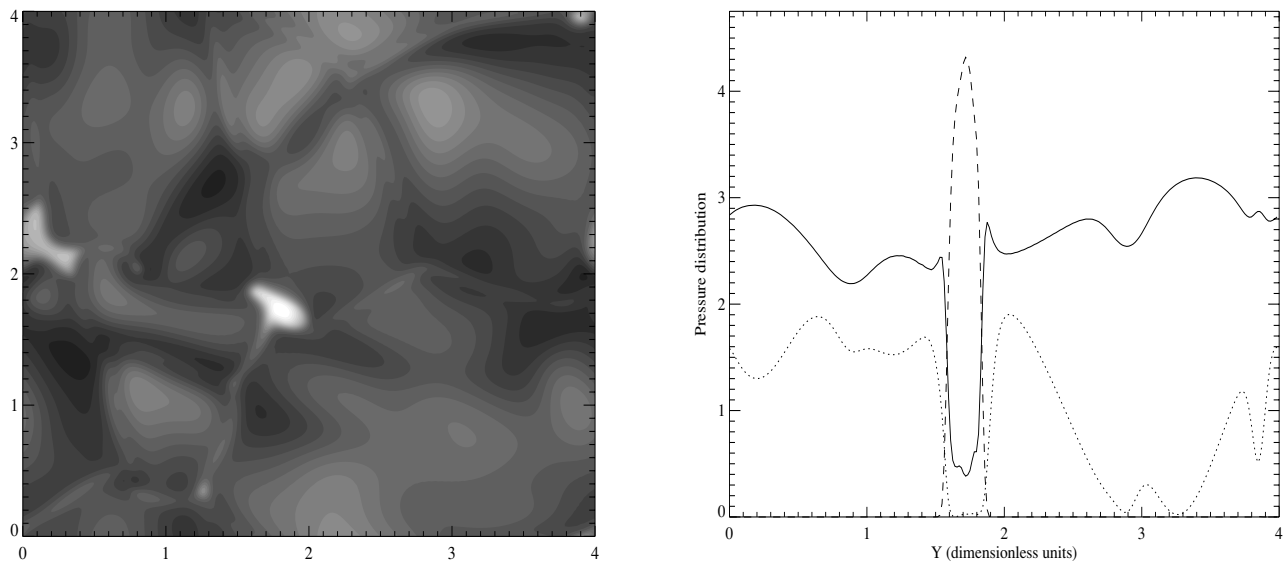

Figure 2. Left: The pressure distribution (gas plus magnetic) at the upper surface of the computational domain. Right: The pressure distribution at the upper surface along a horizontal cut through the strongest magnetic feature. The solid line shows the gas pressure distribution, whilst the dashed line shows the magnetic pressure. The dotted line shows the (so called) "dynamical pressure" $=\rho\left(u^{2}+v^{2}\right)$, which is playing a crucial role in the dynamics.

magnetic pressure within the magnetic regions typically exceeds the gas pressure of the non-magnetic surroundings (as illustrated in figure 2). The so called dynamical pressure (due to the horizontal motions of the surrounding convection) is playing a crucial role in confining these magnetic features to localised regions.

\section{Discussion}

In these (relatively) high magnetic Reynolds number simulations, super-equipartition magnetic features arise naturally as a result of the interaction between convection and magnetic fields. These super-equipartition features are always associated with partially evacuated regions. Idealised models of this process (the so called "convective collapse" instability - see, e.g. Spruit \& Zweibel 1979) assume that the magnetic feature can be modelled as a thin flux tube that is always in total pressure balance (gas plus magnetic) with its surroundings. This simulation has highlighted the dynamical role played by the ambient convection, with the dynamical pressure playing a key role in confining these super-equipartition magnetic features to localised regions.

\section{Acknowledgements}

I would like to thank Steve Houghton, Michael Proctor and Nigel Weiss for their contributions to this project. These calculations were carried out using the CambridgeCranfield High Performance Computing Facility. This work is supported by PPARC.

\section{References}

Bushby, P.J. \& Houghton, S.M. 2005, MNRAS 362, 313

Grossmann-Doerth, U., Schüssler, M., \& Steiner, O. 1998, A\& A 337, 928

Lin, H. \& Rimmele, T. 1999, ApJ 514, 448

Spruit, H.C. \& Zweibel, E.G. 1979, Solar Phys. 62, 15

Stein, R.F. \& Nordlund, A. 2006, ApJ 642, 1246

Weiss, N.O., Proctor, M.R.E., \& Brownjohn, D.P. 2002, MNRAS 337, 293

Vögler, A., Shelyag, S., Schüssler, M., Cattaneo, F., Emonet, T., \& Linde, T. 2005, A $8 A 429$, 335 